Kompass

Ophthalmologie

\section{Bettina Hohberger}

Koordination

«OphthalmoCampus»

\section{Der hintere Augenabschnitt im Fokus}

Liebe Leserinnen und Leser,

ich freue mich sehr, den «OphthalmoCampus» des Kompass Ophthalmologie von Frau Prof. Dr. Joachim übernehmen zu dürfen. Wir werden uns in dieser Rubrik weiterhin mit interessanten und aktuellen Themen aus der Praxis, Klinik, Forschung und Berufspolitik befassen.

Mit der aktuellen Ausgabe starten wir den Beginn einer neuen Reihe im OphthalmoCamus: «Die Basics» werden aktuelle Themen aus der Ophthalmologie thematisieren und werden Ihnen die physikalischen, biochemischen und pathophysiologischen Zusammenhänge und Hintergründe auf dem jeweiligen Gebiet erläutern. Haben Sie Fragen oder möchten Sie etwas aus dem diagnostischen, therapeutischen oder pathogenetischen Bereich erklärt haben? Dann schreiben Sie uns (bettina.hohberger@uk-erlangen.de) - wir werden uns dieser in einer der kommenden Ausgaben von «Die Bascis» widmen. Wir freuen uns über Ihre Zuschriften!

Mit der Rubrik «Blickdiagnose» werden wir Ihnen Krankheitsbilder vorstellen - realitätsgetreu Ihrem Praxis- und Klinikalltag - nach dem Motto: Ein Blick - eine Diagnose!

Die aktuelle Ausgabe setzt den Schwerpunkt auf die hinteren Augenabschnitte: «Die Basics» beginnt Ihre Reihe mit dem physikalischen Background der OCT-Angiographie. Die Rubrik «Blickdiagnose» wird Ihnen einen Patienten mit einem Krankheitsbild aus dem hinteren Augenabschnitt präsentieren. Ein weiterer Beitrag wird Ihnen die RetinaRisk-App vorstellen - speziell entwickelt für Patienten mit Diabetes. Aus aktuellem Anlass haben wir einen Beitrag eines Virologen zu der Thematik «Was ein Augenarzt über das neue Coronavirus wissen sollte» integriert.

Wir wünschen Ihnen viel Freude mit der aktuellen Ausgabe des «OphthalmoCampus»!

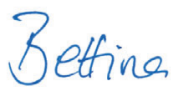

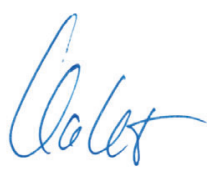

Ihre

Bettina Hohberger 
- Bisher kursierten beim Menschen mindestens vier «endemische» Coronaviren (CoV), die im Klinikbereich für ca. 5-10\% der nachgewiesenen Virusinfektionen verantwortlich waren. Zudem gab es 2002/2003 den Ausbruch des Severe Acute Respiratory Syndrome (SARS)-CoV, und seit 2012 kommt es im mittleren Osten immer wieder zu Erkrankungen am Middle East Respiratory Syndrome (MERS; MERS-CoV). Das neue Coronavirus SARS-CoV-2 ist Erreger der "Coronavirus Disease 2019": COVID-19.

- SARS-CoV-2/COVID-19 stammt aus dem Tierreich und wurde im letzten Quartal 2019 in Wuhan, Provinz Hubei, China, erstmals auf den Menschen übertragen [1]. Genetisch am ähnlichsten ist dem neuen Erreger ein Coronavirus aus Fledermäusen, gefolgt vom Coronavirus der SARS-Epidemie 2003 (SARSCoV) [2].

- Für Aussagen zur tatsächlichen Durchseuchung und über das tatsächliche Erkrankungsrisiko und Gefährdungspotential durch COVID-2019 fehlen noch Seroprävalenzstudien, welche retrospektiv auch asymptomatische Infizierte erkennen können.
- Die Sterblichkeit bei schwerer Erkrankung an dem neuen Virus ist auf jeden Fall deutlich geringer als bei SARS (ca. 10\%) oder MERS (ca. 30\%). Höhere Sterblichkeit wurde bei alten Menschen und bei Menschen mit Grunderkrankungen (Herz-Kreislauf, Lunge, Krebs, Stoffwechsel) beobachtet, Kinder und Jugendliche erkranken hingegen leichter oder bleiben auch asymptomatisch [3]. Die mittlere Inkubationszeit liegt bei etwa 5 Tagen [4], es sind aber bis zu 14 Tagen möglich.

- Es gibt im Vergleich zur echten Virusgrippe (Influenza A- oder B-Virus) einen deutlich höheren Anteil von Erkrankungen mit milder Symptomatik und auch asymptomatischem Verlauf. Dies begünstigt die Verbreitung und verhindert eine effektive Eindämmung. Man geht davon aus, dass bereits 1-2 Tage vor Symptombeginn das SARS-CoV-2 in respiratorischen Sekreten ausgeschieden wird und ein Übertragungsrisiko besteht.

- Ophthalmologische Manifestationen sind bisher nicht im Fokus von COVID-19; Konjunktivitis ist ein mögliches Frühsymptom [5] und auch beim endemischen humanen CoV NL63 beschrieben [6]. Beteiligungen des Sehorgans und der Sehnerven sind bei tierischen CoV bekannt.

\section{Prophylaxe und Hygiene}

- Augenärzte sind hinsichtlich respiratorischer Viren auf Grund des nahen Gesichtskontakts grundsätzlich einem hohen Ansteckungsrisiko durch Tröpfcheninfektion ausgesetzt.

- Hierarchie mit 3 Ebenen von Schutzmaßnahmen (nach [7]):

1) Administrativ (Vorscreening der Patienten, ggf. Verschieben von Terminen und elektiven Eingriffen);

2) Vermeidung von (Micro-) Aerosol-generierenden Prozeduren (kontaktlose Tonometrie, Nasenendoskopie, Allgemeinnarkosen) soweit möglich;

3) Maßnahmen zum Schutz und Infektionskontrolle (Personaltraining, Schutzschilde für Spaltlampen, regelmäßige und häufige Gerätedesinfektion, persönliche Hygiene und Schutzausrüstung (Schutzbrille, -maske))

- Mund-Nasenschutz für Patienten mit Atemwegsinfektionen; FFP2-Maske, Schutzbrille und Schutzkleidung für den Arzt. Ungeschützter naher Kontakt zu einem SARS-CoV- 2-infizierten Patienten, z.B. im Rahmen der augenärztlichen Untersuchung, entspricht einer «Kontaktperson der Kategorie I mit engem Kontakt («höheres» Infektionsrisiko)» mit der wahrscheinlichen Folge einer 14-tägigen häuslichen Absonderung.

- Coronaviren können auf kontaminierten Oberflächen mehrere Tage infektiös bleiben [8]. Coronaviren sind als behüllte Viren empfindlich gegen Detergentien (Seife) und alkoholische Desinfektionsmittel. Desinfektion von Geräten/Oberflächen mit geeigneten sogennanten begrenzt viruziden Desinfektionsmitteln.

- Übliche Hygienemaßnahmen, insbesondere regelmäßiges Händewaschen mit Seife (min. 30 s), Händedesinfektion nach Anwendungsvorschrift, nicht ins Gesicht fassen, gute Husten- und Niesetikette. 


\section{Therapie}

- Therapie bisher nur symptombezogen; in Zellkultur und in Tierversuchen (SARS/MERS-CoV-Modelle) erprobte Wirkstoffe unterdrücken unter Laborbedingungen auch SARSCoV-2 [9]. Verschiedene Phase-I/II-Studien laufen in China.

- Eine breite und rechtzeitige Verfügbarkeit von SARS-CoV2-Impfstoffen binnen Jahresfrist ist nicht zu erwarten. Die Impfstoffentwicklung ist kompliziert, tierexperimentelle
Vordaten (felines Coronavirus) weisen auf ein Risiko antikörpervermittelter Verstärkung bei partieller Immunität [10, 11] hin, ähnlich wie beim Denguevirus [12].

- Die jährliche Influenzaimpfung ist im nächsten Winter besonders wichtig zur Reduktion der allgemeinen Krankheitsbelastung im Gesundheitssystem.

\section{Literatur}

1. Zhu N, Zhang D, Wang W, et al. N Engl J Med. 2020;382:727-733.

2. Zhou P, Yang XL, Wang XG, et al. Nature. 2020;579:270-273.

3. Wu Z, McGoogan JM. JAMA. 2020; DOI:10.1001/jama.2020.2648.

4. Li Q, Guan X, Wu P, et al. N Engl J Med 2020; DOI:10.1056/NEJMoa2001316.

5. Li JO, Lam DSC, Chen Y, Ting DSW. 2020. Br J Ophthalmol 104:297-298.

6. Vabret A, Mourez T, Dina J, et al. Emerg Infect Dis. 2005;11:1225-1229.

7. Lai THT, Tang EWH, Chau SKY, et al. Graefes Arch Clin Exp Ophthalmol. 2020; DOI:10.1007/s00417-020-04641-8.

8. Kampf G, Todt D, Pfaender S, et al. J Hosp Infect. 2020;104:246-251.

9. Wang M, Cao R, Zhang L, et al. Cell Research. 2020;30:269-271.

10. Vennema H, de Groot RJ, Harbour DA, Dalderup M, Gruffydd-Jones T, Horzinek MC, Spaan WJ. 1990. J Virol 64:1407-9.

11. Tetro JA. 2020. Microbes Infect doi:10.1016/j.micinf.2020.02.006.

12. Feinberg MB, Ahmed R. 2017. Science 358:865-866.

\section{Weitere Informationsquellen}

Robert-Koch-Institut

- www.rki.de/DE/Content/InfAZ/N/Neuartiges_Coronavirus/nCoV.html

- www.rki.de/DE/Content/InfAZ/N/Neuartiges_Coronavirus/Massnahmen_Verdachtsfall_Infografik_Tab.html

- www.rki.de/DE/Content/InfAZ/N/Neuartiges_Coronavirus/Hygiene.html

Weltgesundheitsorganisation

- www.who.int/emergencies/diseases/novel-coronavirus-2019

Europäische Seuchenbehörde

- www.ecdc.europa.eu/en

Autor: Prof. Dr. Armin Ensser, Facharzt für Mikrobiologie, Virologie und Infektionsepidemiologie, Virologisches Institut, Universitätsklinikum Erlangen, Schlossgarten 4, 91054 Erlangen, Deutschland

\section{Blickdiagnose}

\section{Welche Diagnose vermuten Sie?}

Vor 12 Jahren stellte sich ein damals 18-jähriger junger Mann mit seit der Kindheit bestehenden Dunkelsehproblemen in der Spezialsprechstunde für seltene Netzhauterkankungen vor. Die Sehschärfe war beidseits gut erhalten $(R / L 1,0)$, jedoch belasteten zunehmende konzentrische Gesichtsfelddefekte. Das durchgeführte

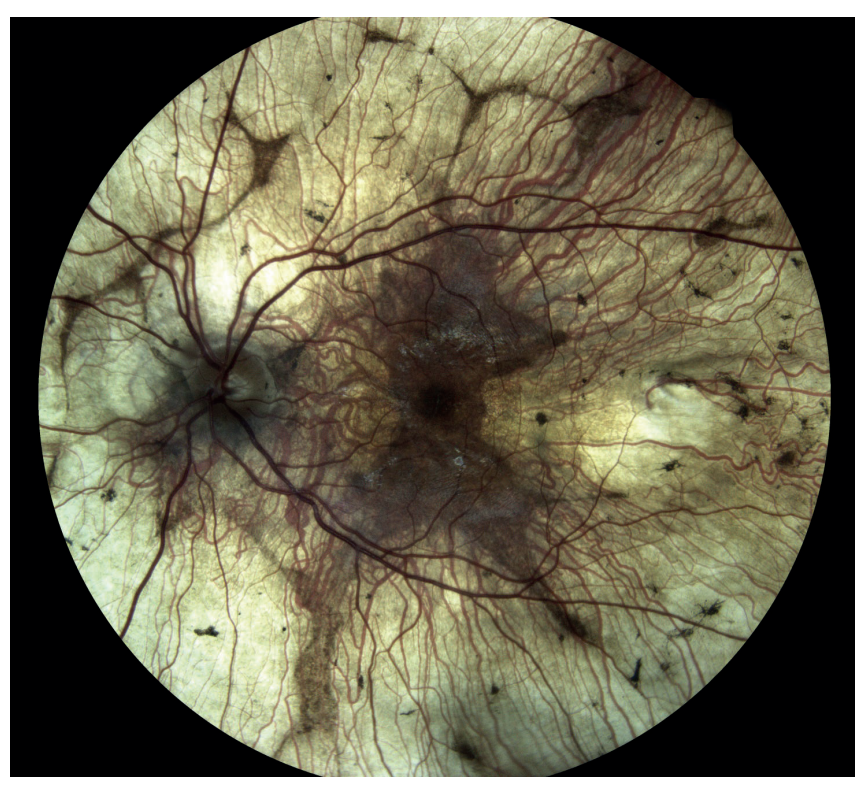

Ganzfeld-ERG war photopisch und skotopisch ausgelöscht. Der Patient gab einen im frühen Erwachsenenalter erblindeten Großvater mütterlicherseits an. Weitere Erkrankte ergab die Familienanamnese nicht, im Speziellen waren beide Eltern sehgesund, jedoch zeigte eine bei der Mutter durchgeführte Untersuchung ein auffälliges, radiäres Mosaik in Funduskopie und Fundusautofluoreszenz.

Im Verlauf kam es zu einem Voranschreiten der chorioretinalen Atrophie (siehe Abbildung) und einer zentralen Involvierung mit partiellem Bandenverlust der äußeren neurosensorischen Netzhaut. Der Visus reduzierte sich auf 0,63 und die Einschränkungen im Alltag nahmen zu. Seit zwei Jahren nimmt der Patient an einer multizentrischen Gentherapiestudie teil.

\section{Auflösung unter: \\ www.karger.com/blickdiagnose-kop-2-2020}

Kontakt: Dr. Philipp Herrmann, PhD, FEBO, Oberarzt und Leiter der Sprechstunde für seltene Netzhauterkankungen, Universitäts-Augenklinik Bonn, Ernst-Abbe-Straße 2, 53127 Bonn, Deutschland, philipp.herrmann@ukbonn.de

Aktuelles Fundusfoto des linken Auges. Zu erkennen die zentrale, sternförmige Restinsel intakter Netzhaut mit umgebender chorioretinaler Atrophie, welche den Blick auf die choroidalen Gefäße und Sklera freigibt. 


\section{Die Basics}

\section{OCT-Angiographie - der physikalische Hintergrund}

Die optische Kohärenztomographie (OCT) [1] ist seit Jahren ein in der Augenheilkunde etabliertes Bildgebungsverfahren, da sie nicht invasiv ist und in vivo ein 3-dimensionales Volumen des Gewebes der Retina aufnehmen kann. Dazu wird ein Lichtstrahl auf einen Punkt der Objektoberfläche abgegeben, welcher mehrere Millimeter in das Gewebe eindringen kann. Aufgrund der unterschiedlichen optischen Eigenschaften des Gewebes wird dieser Lichtstrahl in der Tiefe unterschiedlich (teil-)reflektiert. Das reflektierte Licht, welches wieder an der Oberfläche ankommt, kann mit Hilfe einer interferometrischen Messung detektiert werden. Somit kann ein Tiefenprofil des reflektierten Lichts erstellt werden (Abb. 1). Um ein Schnittbild der Retina zu erhalten, werden mehrere Tiefenprofile nebeneinander aufgenommen. Analog zur Ultraschallbildgebung wird auch bei dem OCT das Tiefenprofil als «A-Scan» bezeichnet und das Schnittbild als «B-Scan». Werden nun mehrere B-Scans hintereinander aufgenommen, entsteht ein 3-dimensionales Volumen des Objekts.

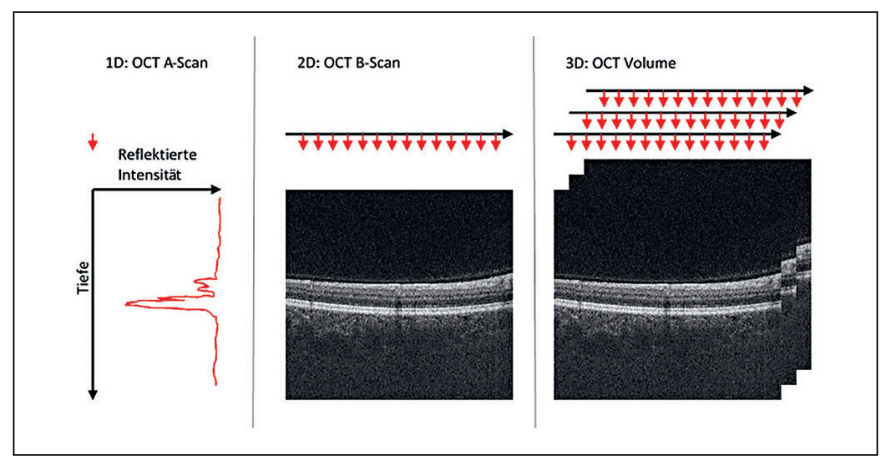

Abb. 1. OCT Datenaufnahme: Für ein Tiefenprofil (A-Scan) wird das reflektierte Licht an einer Stelle des Gewebes gemessen. Um ein Schnittbild (B-Scan) zu erlangen, wird die Tiefenmessung an mehreren benachbarten Punkten aufgenommen. Um ein Volumen zu erhalten, werden mehrere B-Scans an verschiedenen Stellen des Gewebes aufgenommen.

Im Gegensatz zu den Gewebestrukturen der Retina können deren Blutgefäße allerdings nur schlecht dargestellt werden, da sich die Gefäßwände und das darin enthaltene Blut in ihren optischen Eigenschaften nur wenig von den umgebenen Strukturen unterscheiden. Deswegen wurde eine Erweiterung des OCT, die sogenannte OCT-Angiographie (OCT-A) entwickelt, welche anhand von OCT B-Scans Informationen über den Blutfluss berechnen kann. In der OCT-A werden zusätzlich zu den strukturellen Unterschieden auch zeitliche Unterschiede im Reflexionsverhalten erfasst. Hier verwendet man die Idee, dass in einem OCT B-Scan die stationären Gewebeanteile das Licht gleich reflektieren und somit auch im Bild gleich erscheinen. Herrscht allerdings Bewegung in einem Teil des Gewebes, wie es bei den Blutplättchen in den Gefäßen der Fall ist, dann befinden sich diese bewegten Strukturen in zwei Aufnahmen an leicht unterschiedlichen Orten im Bildfeld und reflektieren somit auch das Licht unterschiedlich. Diese Veränderung der Intensität kann mit mehreren Algorithmen entdeckt werden [2], anhand de- rer dann ein OCT-A B-Scan (Abb. 2b) berechnet wird. Um einen OCT-A B-Scan zu berechnen, sind folglich nur zwei aufgenommene OCT B-Scans an derselben Stelle im Gewebe notwendig. Dadurch ist auch die OCT-A nicht invasiv und es wird kein Kontrastmittel benötigt. Dieser OCT-A B-Scan ist allerdings schwer zu interpretieren, da er die Gefäße nur im Querschnitt zeigt. Deswegen wird meistens eine sogenannte «En-face»-Projektion dargestellt (Abb. 2c).
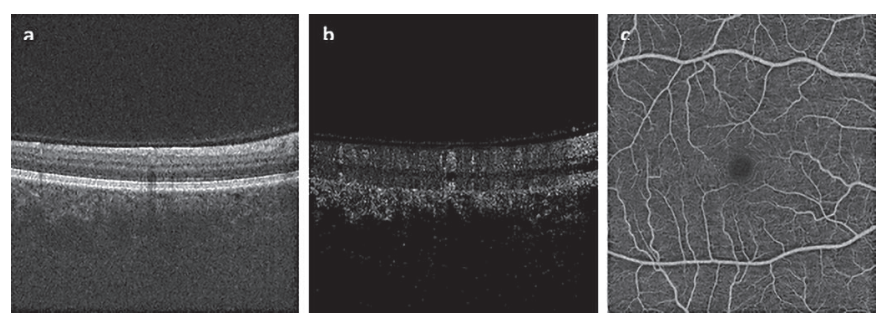

Abb. 2. Aus zwei hintereinander aufgenommen OCT B-Scans (a) kann durch die Analyse der Intensitätsdifferenzen ein OCT-A B-Scan (b) berechnet werden. Da dieser allerdings schwer zu interpretieren ist, wird oft eine En-face-Projektion dargestellt.

Um eine En-face-Projektion zu erstellen, werden aus dem berechneten OCT-A Volumen eine oder mehrere Tiefenschichten ausgewählt und z.B. gemittelt (Abb. 3). Dies hat eine gewisse Ähnlichkeit zur Fluoreszenzangiografie.

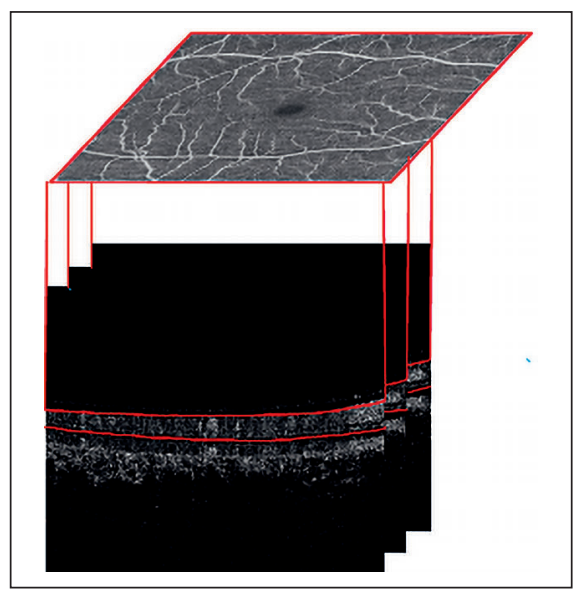

Aus einen OCT-A Volumen werden eine oder mehrere Retinaschichten ausgewählt und diese z.B. gemittelt, um eine En-face-Projektion zu erhalten.

\section{Literatur}

1. Huang D, Swanson EA, Lin CP, et al.: Optical coherence tomography. Science. 1991; 254:1178-1181.

2. Ploner SB, Riess C, Schottenhamml J, et al.: A joint probabilistic model for speckle variance, amplitude decorrelation and interframe variance (IFV) optical coherence tomography angiography. Bildverarbeitung für die Medizin 2018. Springer Vieweg, Berlin, Heidelberg, 2018, pp. 98-102.

Autorin: Julia Schottenhamml, Pattern Recognition Lab, Friedrich-Alexander-Universität Erlangen-Nürnberg 


\section{Mobile Health}

\section{Die frühe Diagnostik ist der Schlüssel!}

Die diabetische Retinopathie ist weltweit eine der Hauptursachen für eine Erblindung im erwerbsfähigen Alter. Diese schwerwiegende Erkrankung zeigt normalerweise keine Symptome in den frühen Stadien, was bedeutet, dass sich der Patient häufig schon in einem fortgeschrittenen Stadium der Erkrankung befindet, wenn Symptome wahrnehmbar werden und er in der augenärztlichen Praxis vorstellig wird. Die frühzeitige Diagnostik und Behandlung der diabetischen Retinopathie kann in 95\% der Fälle verhindern, dass ein Diabetiker einen Sehverlust und eine eventuelle Erblindung erleiden wird. Früherkennung und Prävention sind jedoch nur dann möglich, wenn die Millionen von Menschen, die weltweit an Diabetes leiden, über die Bedeutung von Augenuntersuchungen informiert werden und dann auch auf ihre Untersuchungsergebnissen entsprechend reagieren.

Die globale Diabetes-Epidemie hat sich seit dem Jahr 2000 weltweit verdreifacht. Es wird damit gerechnet, dass 2045 die Zahl der betroffenen Weltbevölkerung über 600 Millionen betragen wird. Zwei Drittel aller Diabetiker entwickeln im Verlauf ihrer Erkrankung eine diabetische Retinopathie, die Hälfte davon erkrankt innerhalb von 20 Jahren an einer das Augenlicht bedrohenden Verlaufsform. Diese Patienten haben ein hohes Risiko für Sehstörungen oder Erblindung, wenn ihre diabetische Retinopathie nicht rechtzeitig diagnostiziert und behandelt wird.

In mehreren Ländern wurde ein systematisches Augenscreening eingeführt, z.B. im Vereinigten Königreich. Kürzlich wurde veröffentlicht, dass die diabetische Retinopathie nicht mehr die Hauptursache für Erblindung in der britischen Bevölkerung im erwerbsfähigen Alter ist. Leider lässt nur ein kleiner Teil der derzeit ca. 463 Millionen Diabetiker regelmäßig seine Augen untersuchen. Dies kann auf die Nichtverfügbarkeit von Augenuntersuchungsmöglichkeiten zurückzuführen sein. Aber auch wenn strenge Augenuntersuchungsprogramme existieren, ist das Nichteinhalten der Untersuchungen und Therapien ein ernstes Problem.

Was wäre nötig, um sicherzustellen, dass jede Person, die mit Diabetes lebt, aufgrund ihres Risikoprofils Zugang zu regelmäßigen Augenuntersuchungen hätte? Neue Technologien und eine verstärkte Betonung von personalisierten Ansätzen würden helfen. Die meisten aktuellen Screening-Programme verwenden Farbfotografien des Fundus. Eine Auswertung dieser Aufnahmen und die Diagnostik einer diabetischen Retinopathie erfordert speziell geschultes Personal. In den letzten Jahren wurden neue Bildgebungsmodalitäten eingeführt, die die Genauigkeit der Diagnose, die Untersuchungszeit am Patienten und den Kostenfaktor erheblich verbessert haben. Smartphone-basierte Fundusfotografie, optische Kohärenztomographie der Makula, Ultra-Weitwinkel Bildgebungstechnologien und das Auswerten der Aufnahmen unter Nutzung künstlicher Intelligenz sind einige der neuen Technologien, die sich mit den Einschränkungen der aktuellen Ansätze befassen und versuchen diese zu lösen.

Der klinisch validierte RetinaRisk-Algorithmus ist eine dieser Technologien, die einen erheblichen Beitrag zur Früherkennung einer diabetischen Retinopathie, zu einer Beschleunigung/Erleichterung von Augen-Screening-Programmen und zur verbesserten Aufklärung über die diabetische Retinopathie leisten. Mit der RetinaRiskApp, die für alle Smartphones kostenlos verfügbar ist, können Diabetiker ihr individuelles Risiko für die Entwicklung einer die Sehfähigkeit bedrohenden diabetischen Retinopathie anhand ihres Risikoprofils bewerten und überwachen. Hierzu geben die Benutzer Informationen zu ihrem Geschlecht, der Art und Dauer ihres Diabetes, ob bereits eine Retinopathie diagnostiziert wurde, ihren Langzeit-Blutzuckerspiegel (HbA1c) und ihren Blutdruck ein. Basierend auf diesen Parametern berechnet die App das jeweilige individuelle Risiko an einer diabetischen Retinopathie zu erkranken und veranschaulicht dieses anhand einer farbbasierten Skala (grün für geringes Risiko, gelb für mittleres Risiko, rot für hohes Risiko). Mit der Analysetaste können die Benutzer sich auch anzeigen lassen, welches ihre Hauptrisikofaktoren (HbA1c oder Blutdruck) sind und wie diese gemindert werden können, um das Risiko einer Sehbehinderung zu verringern. Die einfache und anschauliche Risikoberechnung durch die RetinaRisk-App zeigt dem Patienten deutlich, wie eine Verbesserung seiner Risikofaktoren an einer potenziell zur Erblindung führenden diabetischen Retinopathie realisiert werden kann, was zudem auch die langfristigen Kosten für medizinische Diagnostik und Therapie reduzieren könnte.

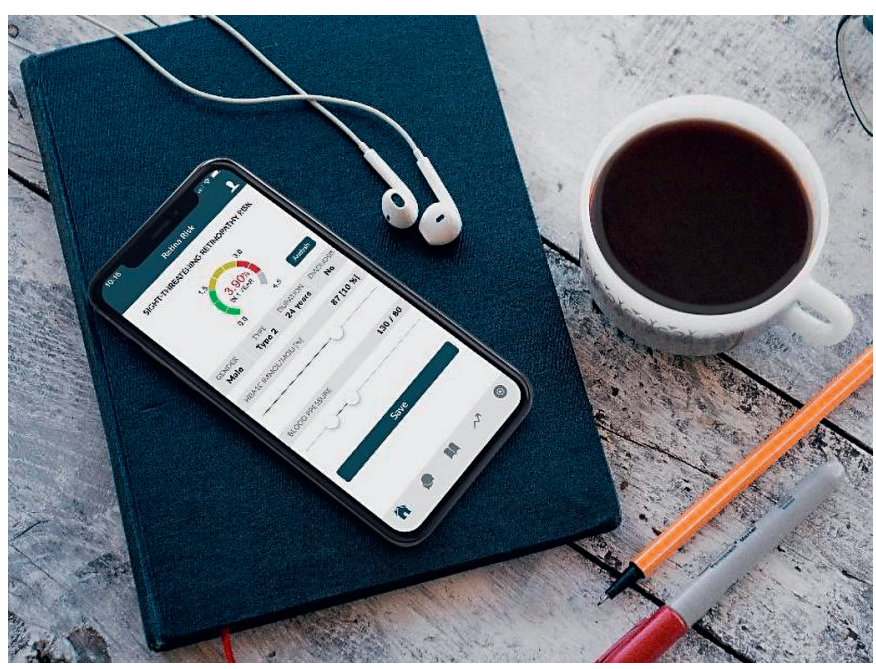

Abb. 1. Die Benutzeroberfläche der Retina Risk App.

Wenn sich Diabetiker ihres eigenen Risikos bewusst sind, könnten sie Maßnahmen ergreifen (z.B. frühzeitige Behandlung und Änderung des Lebensstils), um das Erkranken an bzw. das Fortschreiten einer diabetischen Retinopathie zu verhindern/verlangsamen. Dies würde zum einen die Lebensqualität der weltweit 463 Millionen Diabetiker erhalten bzw. langfristig verbessern, zum anderen auch die Kosten des Gesundheitssystems, die für die Behandlung aufgebracht werden müssten, reduzieren.

Die RetinaRisk-App enthält detaillierte Richtlinien und nützliche Informationen über Diabetes und diabetische Retinopathie allge-
92

Kompass Ophthalmol 2020;6:88-93 DOI: $10.1159 / 000507770$ 
mein. Darüber hinaus gibt sie Tipps zu einer verbesserten Selbstversorgung, die es den Benutzern ermöglichen, ihren Zustand besser zu verstehen und sie auch dazu motivieren, aktiv einen Beitrag für ihre eigene Gesunderhaltung zu leisten. Auch ermöglicht die App Diabetikern sich stärker an den Entscheidungen zu beteiligen, die ihre Gesundheit betreffen. Darüber hinaus unterstreicht sie die Bedeutung regelmäßiger Augenuntersuchungen und rechtzeitiger medizinischer Therapien.

Die RetinaRisk-App wurde von einem in Island ansässigen Unternehmen entwickelt, das von Prof. Dr. Einar Stefansson, Chefarzt der Universitäts-Augenklinik Reykjavik, und Dr. Arna Gudmundsdottir, Endokrinologin am Nationalen Krankenhaus in Reykjavik, gegründet wurde. Beide haben mehr als 30 Jahre Erfahrung in der Diagnostik und Behandlung von Patienten mit diabetischer Retinopathie und der Behandlung des Diabetes mellitus.

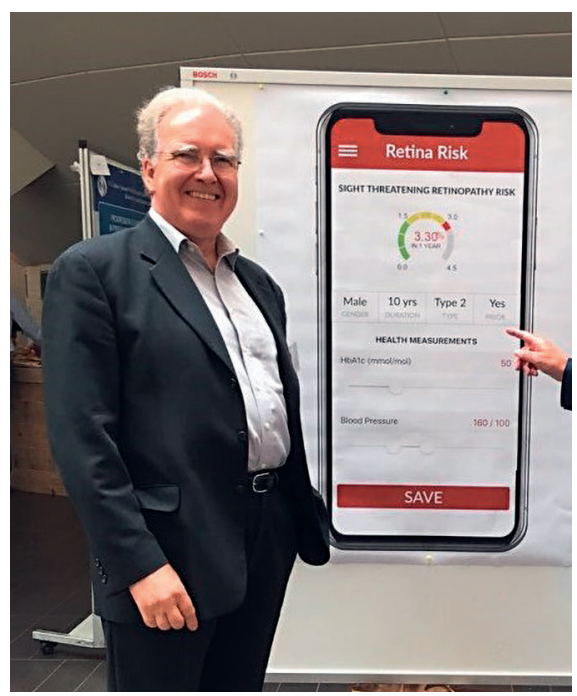

Prof. Einar Stefansson

Der Algorithmus der RetinaRisk-App basiert auf umfangreichen internationalen Untersuchungen zu Risikofaktoren, die bekanntermaßen das Fortschreiten einer diabetischen Retinopathie beeinflussen. Die klinische Validierung an über 20000 Diabetikern gilt als zuverlässig, die Ergebnisse wurden bereits in mehreren medizinischen Fachzeitschriften veröffentlicht (z.B. frei verfügbar: https://ocimumpublishers.com/journal/eye-study-treatment/early-online/a-smartphoneapplication-for-personalized-approach-to-diabetic-eye-disease).
Für den Zugriff auf persönliche Gesundheitsdaten wird die RetinaRisk-App von der digi.me-Plattform unterstützt. Das bedeutet, dass keine gesundheitsbezogenen Daten von RetinaRisk gespeichert werden. Die kostenlose RetinaRisk-App kann über folgende Links heruntergeladen werden:

Google Play:

https://play.google.com/store/apps/details?id=com.retinarisk Apple Store:

https://itunes.apple.com/us/app/retinarisk/id1370996145?mt=8

Neue Technologien, wie die RetinaRisk-App können den globalen Kampf gegen die vermeidbare Blindheit durch Diabetes erheblich beeinflussen, sie können zudem zu einer integrierten, auf den individuellen Patienten ausgerichteten ophthalmologischen Therapie beitragen, was darüber hinaus die Gesundheitssysteme stärken und die Bedürfnisse der Bevölkerung erfüllen würde, wie dies im «World Vision Report», der kürzlich von der WHO veröffentlicht wurde, gefordert wird (www.who.int/publications-detail/world-reporton-vision).

Die RetinaRisk-App ist für alle Smartphones kostenlos verfügbar. Mit der App kann jeder Patient sein persönliches Risiko für die Progression einer zu Sehbehinderungen führenden diabetischen Retinopathie abschätzen. Die App informiert über die diabetische Retinopathie und Risikofaktoren, motiviert und informiert den Patienten, sein Risikoprofil zu ändern und zu verbessern. Die App ist ein Schritt in die Richtung, das Verständnis der Patienten individuell durch die validierte Interpretation der verfügbaren klinischen Daten zu verbessern: Was bedeuten diese Daten für jeden Einzelnen, wie können die Risikofaktoren zur Risikominderung modifiziert werden? Die App bietet eine persönliche Prognose für das Fortschreiten der Retinopathie. Mehr Informationen zur RetinaRiskApp: www.retinarisk.com.

Autoren: Sibylle Scholtz ${ }^{\mathrm{d}}$, Thor Aspelund ${ }^{\mathrm{b}}$, Stefán Einarsson ${ }^{\mathrm{a}}$, Arna Gudmundsdottirc, Sigurbjörg Jonsdottira, Aegir Thor Steinarssonª, Einar Stefansson $^{a}$

a Risk ehf, Reykjavik, Island; ' Universität Island, Reykjavik, Island, ' Landspitali, Universitätsklinik, Reykjavik, Island; ${ }^{d}$ Institut für Experimentelle Ophthalmologie, Universität des Saarlands, Homburg/Saar, Deutschland

Kontakt: Dr. Sibylle Scholtz, Sibylle.scholtz@gmx.de 